ELUA

ISSN 2171-6692

Núm. 36, 2021, págs. 129-141

https://doi.org/10.14198/ELUA.2021.36.07

\title{
Aproximación al tratamiento de las paremias como pragmatemas y sus posibles aplicaciones
}

Approach to the study of paroemias as pragmatemes and their possible aplications

\author{
Almudena Caño Laguna \\ Universidad Complutense de Madrid, España \\ almcano@ucm.es \\ https://orcid.org/0000-0002-8953-2569
}

\section{Resumen}

Las paremias se definen como expresiones que representan un conocimiento social o cultural compartido en la comunicación y se pueden manifestar de diferentes formas, entre otros en refranes, proverbios y aforismos. Por definición, las diferentes representaciones paremiológicas se asocian a la comunicación o a la cadena discursiva, pero el trabajo fraseológico $\mathrm{y}$ algunas de sus aplicaciones abandonan esta visión para enfocarla al estudio de su composición formal o a su representación en la literatura. No abundan los enfoques basados en el contexto comunicativo, a pesar de que resultan especialmente necesarios en la enseñanza en secundaria o en la Enseñanza de Español como Lengua Extranjera (E/LE). Este trabajo pretende sumar en ese sentido con un estudio paremiológico desde un enfoque basado en la Teoría Sentido-Texto. Este modelo permite asociar algunas paremias a determinados contextos extralingüísticos bajo el concepto de pragmatema y en nuestro trabajo hemos que-

\begin{abstract}
Paroemias are defined as expressions representing a shared knowledge in communication that take on many different forms, such as sayings, proverbs or aphorisms, among others. By definition, paremiological representations are associated with discourse and communication. However, studies on phraseology and some of its implementations focus rather on the form of paroemias or their depiction in literature. This work aims at addressing the study of paroemias from the approach of the Meaning-Text Theory, according to which lexical constructions can be associated with given extralinguistic contexts under the term pragmateme. Thus, paroemias might be considered pragmatemes. As a consequence, paremiologic applications (e.g. teaching Spanish as a Foreign Language (EL2/EFL) or secondary education) might be approached from a perspective based on the communicative act.

To check for the appropriateness of the associations between paroemias and their context
\end{abstract}

Para citar este artículo: Caño Laguna, A. (2021). Aproximación al tratamiento de las paremias como pragmatemas y sus posibles aplicaciones. ELUA, (36), 129-141. https://doi.org/10.14198/ ELUA.2021.36.07

Recibido: 31/07/2021, Aceptado: 04/10/2021

C) 2021 Almudena Caño Laguna

Este trabajo está sujeto a una licencia de Reconocimiento 4.0 Internacional de

Creative Commons (CC BY 4.0) 
rido averiguar qué condiciones son necesarias para que esta asociación tenga lugar,

Para comprobar que las asociaciones entre las paremias y sus contextos de uso son apropiadas, se realizan tres pruebas a un total de 154 informantes, todos ellos hablantes de español como lengua materna. En estas pruebas se extraen las paremias más representativas en el conocimiento colectivo, se asocian tanto a definiciones extraídas de diferentes diccionarios fraseológicos como a diferentes contextos relacionados con la interpretación de la propia paremia y con las restricciones de uso según el grado de confianza en la relación jerárquica entre los hablantes. También se tienen en cuenta en el análisis estadístico variables independientes como la edad y el lugar de procedencia de los hablantes, además de la frecuencia de uso de paremias en la conversación.

Con los resultados obtenidos, se puede considerar que algunas de esas paremias pueden ser consideradas pragmatemas y, por lo tanto, pueden modificarse sus aplicaciones basándolas en el conocimiento contextual. Además, estos resultados permiten ampliar los horizontes de estudio, pudiendo analizar las paremias desde perspectivas contextuales y, a su vez, dando lugar a una clave para profundizar en el concepto pragmatema.

PALABRAS CLAVE: paremia; pragmatema; fraseología; ELE; educación secundaria. of use, three tests were administered to a total of 154 participants, all of them Spanish native speakers. The most representative paroemias in the shared knowledge are extracted from these tests, where paroemias are associated with definitions extracted from several phraseologic dictionaries. They are also associated with different contexts related to the interpretation of the paroemia itself and with the use restrictions, according to the degree of confidence in the hierarchical relationship among speakers. Furthermore, statistical analyses considered independent variables such as the participants' age and the place of residence, together with the frequency of use of paroemias in conversation.

Results suggest that some paroemias might be considered pragmatemes and, therefore, their use applications might be modified based on contextual knowledge. Moreover, results allow to broaden research horizons (since paroemias can be analyzed from a different perspective) and provide a key to deepen into the concept of pragmateme.

KEY WORDS: paremiology; phraseology; pragmateme; EFL; secondary education.

\section{ESTADO DE LA CUESTIÓN}

\subsection{Paremias: terminología y definición}

El presente estudio sobre las paremias del español se aborda desde el ámbito de la lexicografía, en concreto, desde la Teoría Sentido-Texto, modelo en el que se analizan con detalle las relaciones léxicas y semánticas (Melčuk 1996; Polguère 2011).

Por un lado, se podría definir la paremia como una expresión estereotipada que representa un saber compartido que se puede manifestar con refranes populares o proverbios, insertos o no en una cadena discursiva (Anscombre 1997). Como se adelanta en dicha definición, las paremias pueden representarse de diferentes modos, aunque en muchas ocasiones los límites entre sus términos se difuminan. Tal es la dificultad, que se han desarrollado diversas clasificaciones en función de diferentes parámetros, como la fijación combinatoria de los componentes (Zuluaga 1980), criterios de funcionalidad (Corpas Pastor 1996), o 
la fijación sintáctica y semántica (Solano Rodríguez 2012). El trabajo de Crida Álvarez y Sevilla Muñoz (2015) realiza una clasificación en función del origen y del registro de uso, criterios más cercanos a una perspectiva comunicativa. En concreto, los autores diferencian aquellas paremias de origen conocido y uso culto, bajo las que se enmarcan los proverbios y los aforismos; y las de origen anónimo y uso popular, como es el caso de los refranes, las frases y locuciones proverbiales y los dialogismos. De acuerdo con su clasificación, el objeto de estudio de este trabajo se ubica entre las paremias del segundo tipo. Más detalladamente, nuestro objeto de estudio es el de los refranes que exigen un contexto extralingüístico específico y que, por consiguiente, pueden ser etiquetados como pragmatemas.

Desde su enfoque lexicológico y lexicográfico, la Teoría Sentido-Texto se ofrece como un modelo en el que se estudian las limitaciones del significado de las palabras y de las unidades fraseológicas. En esta perspectiva se define el concepto pragmatema como un tipo de formulema que cuenta con una delimitación pragmática; es decir: un enunciado que contiene un referente abstracto que se emplea en contextos concretos (Melčuk 2015). Es el caso de ¡feliz cumpleaños!, fórmula que necesita de la condición contextual de la celebración de un cumpleaños.

Blanco (2014) describe los pragmatemas desde el punto de vista lexicográfico y se sirve de una macroestructura que se adapta a unidades frásticas que están condicionadas por una situación, entre las que se diferencian unidades monoléxicas (formadas por una sola palabra, como ¡hola!) y unidades pluriléxicas (como ¡buenas tardes!). Muchos pragmatemas son fórmulas rutinarias que se asocian a situaciones concretas. Entre otros autores, Barrios Rodríguez (2017) ha planteado el concepto de pragmatema en un sentido más amplio, considerando que pueden ser pragmatemas algunos clichés, como los formulemas, pues son dependientes del contexto extralingüístico. La autora observa, además, que no forman necesariamente estructuras composicionales.

Aquí entendemos por paremia una expresión de uso popular, que los hablantes de una lengua son capaces de emplear en una conversación. La pregunta de investigación plantea la posibilidad de relacionar estas expresiones con el concepto de pragmatema en sentido amplio y, en caso de que la respuesta sea afirmativa, cómo se podrían enseñar las condiciones extralingüísticas de uso de cada refrán a estudiantes de secundaria o de E/LE.

\subsection{Aplicaciones didácticas de las paremias}

Las paremias son un elemento de la comunicación que los hablantes han aprendido, bien mediante la retención memorística, bien basándose en la propia experiencia comunicativa, tarea sencilla debido al extrañamiento que produce el primer contacto del hablante con una paremia en una conversación (Díaz Pérez 1997).

Como consideración más general, se asocian las paremias al conocimiento cultural de una sociedad, de modo que pueden ser consideradas parte de la comunidad linguocultural nacional (Vyshnya, Sevilla Muñoz y Gutthy 2007), es decir, las paremias forman parte de aquellos objetos culturales que se representan en lenguaje. En relación con esta idea, se plantea la necesidad de conocer estos objetos culturales al introducirse en una comunidad de habla. Con el fin de considerar cuáles son aquellas paremias que son necesarias conocer para formar parte de dicha comunidad, algunos investigadores han procurado realizar un acercamiento a un número representativo de paremias que son conocidas en una comuni- 
dad, lo que se ha denominado mínimo paremiológico. Para la lengua española, el trabajo de Tarnovska (2005) consigue llevar a este mínimo a un total de 27 paremias, que más tarde amplía a un total de 250 , que incluye en un diccionario para facilitar el acceso de los hablantes a este conocimiento.

A continuación, resumimos las aportaciones de algunas aplicaciones concretas en las que se trabajan las paremias en la actualidad.

\subsubsection{Enseñanza de español como lengua extranjera (ELE)}

Según el Marco Común Europeo de Referencia (MCER), una de las competencias que los aprendientes deben adquirir es la competencia sociolingüística, en la que se incluyen las expresiones de sabiduría popular. De este modo, los estudiantes de español como lengua extranjera experimentan un acercamiento al conocimiento cultural y social a través de sus representaciones en la lengua. Por otro lado, en el Plan Curricular del Instituto Cervantes (PCIC) el conocimiento fraseológico aparece en los apartados "Referentes culturales", "Saberes y comportamientos socioculturales" y "Habilidades y actitudes interculturales". En todos ellos se dota al hablante de capacidad de reconocimiento de estas expresiones, una aproximación a la sabiduría popular y la posibilidad de conocer elementos culturales a través de ellos, pero, pese al carácter comunicativo del PCIC, esta materia no se presenta como un elemento comunicativo reconocible en diferentes contextos.

El trabajo de Penadés Martínez (1998) resume algunas propuestas realizadas sobre el tratamiento de las paremias en la clase de ELE. En ellas se destaca el uso social que se realiza de las paremias en la comunicación pues, en opinión de la autora, permiten adquirir un discurso más natural, espontáneo y semejante al de un nativo. Si se sigue este razonamiento, se podría concebir el diseño de actividades relacionadas con la paremiología como un acercamiento por niveles al conocimiento del significado de estas expresiones.

Por otro lado, si se observa cuáles son las paremias que comúnmente se emplean en la enseñanza de español como segunda lengua, se pueden encontrar diferentes puntos de vista en su selección. Así lo refleja Leal Riol (2013) al mencionar los consejos que suele dar al profesorado cuando se plantean qué refranes elegir, pues recomienda considerar la variedad de estructuras internas de las paremias, el reflejo cultural o la temática léxica. Algunos otros criterios mencionados por este autor, sí se relacionan con su empleo comunicativo, como son la frecuencia de uso, el factor cultural, o la rentabilidad y productividad.

\subsubsection{Educación secundaria obligatoria (ESO)}

Otro ámbito de enseñanza en el que se pueden desarrollar las paremias es en el currículo de educación secundaria. Concretamente en el currículo de la asignatura de Lengua Castellana y Literatura de la Comunidad de Madrid (BOCM, Decreto 48 2015) se especifica la enseñanza de refranes como parte del Bloque 3, en el apartado relacionado con el manejo de diccionarios, que tiene como fin el aprendizaje de búsqueda de información. Al igual que en el apartado anterior, bajo la premisa de la LOMCE, el Parlamento Europeo propone la adquisición de unas competencias clave en la educación secundaria, entre las que se encuentra la competencia lingüística. Esta competencia se descompone en subcompetencias, entre las que se encuentra la competencia comunicativa. Algunos autores como Hymes y Bernal 
(1996) asumen una vertiente más pragmática de esta competencia lingüística relacionada con el uso del lenguaje en su contexto, lo que permite el trabajo de la lengua en un contexto comunicativo con actividades adecuadas (Lomas y Osoro 1994; Beghadid 2013,). De este modo, es posible hablar de competencias específicas para cada uso del lenguaje, como es el caso de la competencia paremiológica, de Díaz Pérez (1997) y la fraseológica, trabajada por Solano Rodríguez (2007).

Algunas propuestas didácticas para la enseñanza fraseológica, siguiendo con estos criterios, los desarrolla Fernández Pampín (2012), quien propone actividades en las que se entablan relaciones entre la construcción fraseológica y su significado, comparaciones entre refranes de diferentes lenguas, asociaciones entre los refranes y su campo semántico, o la identificación de figuras retóricas.

Como se puede apreciar por las referencias mencionadas, con respecto a la enseñanza de las paremias los profesores se encuentran en primer lugar con el obstáculo de cuáles elegir, y además con el de qué actividades desarrollar para que el estudiante no solo sea capaz de comprenderlas sino, sobre todo, de usarlas correctamente.

\section{METODOLOGÍA, ANÁLISIS Y RESULTADOS}

\subsection{Objetivo, metodología e hipótesis de trabajo}

El interés principal de este trabajo es verificar si algunos refranes se pueden asociar a contextos comunicativos concretos y, en caso afirmativo, realizar propuestas válidas para su enseñanza.

Hemos de mencionar que en este trabajo no buscamos una descripción lexicológica ni lexicográfica de las paremias, ni un estudio de su forma, sino una descripción de los contextos extralingüísticos que acompañan a su uso. Queremos averiguar también la frecuencia de uso, pues consideramos que, como hemos adelantado, debemos empezar enseñando los más frecuentes. Por último, nos interesa conocer con bastante detalle las restricciones de uso.

Para lograr nuestros objetivos, hemos realizado diferentes encuestas que aportarán los datos cuantitativos y cualitativos necesarios para el análisis.

Elaboramos una primera prueba piloto en la que participaron de forma oral diez informantes. En ella se les preguntaba acerca del significado, contexto de uso y situación extralingüística de veintidós paremias. Con los resultados obtenidos en esta prueba, se realizó una segunda encuesta (que a partir de ahora se denominará encuesta de reconocimiento) a un total de cien informantes mediante la plataforma formularios de Google, en la que figuraban las paremias que habían obtenido una frecuencia mayor de aparición en la encuesta anterior. En esta segunda encuesta se proponían no solo los refranes seleccionados junto con las definiciones aportadas por los mismos encuestados, sino también las definiciones ofrecidas por Junceda (1998) y Doval (1997). Se aportaba más de una y se invitaba al informante a escoger una, la que consideraban más apropiada. Junto a las definiciones se ofrecían diferentes contextos extralingüísticos asociados a su uso, entre los que debían escoger cuál era el adecuado para cada refrán.

Por último, se realizó una tercera encuesta a cincuenta informantes (a partir de ahora, encuesta de acceso) en la que invertimos el sentido de las preguntas: si antes les mostrábamos un refrán y les proponíamos la selección de un significado o de un contexto extralingüístico, 
ahora ofrecíamos el contexto y la intención comunicativa, y les pedíamos que escribieran qué refrán dirían en ese caso. Ya no nos interesaban los datos descriptivos de uso, (entre ellos, por ejemplo, a quién se dirige el refrán), o la manera de enunciarlo, sino el acceso a la información paremiológica de los hablantes, es decir, la capacidad de responder con una paremia a una situación comunicativa concreta. Se tuvo en cuenta la diferencia de Tarnovska (2005) entre reconocimiento paremiológico de las paremias (conocimiento pasivo) y disponibilidad (conocimiento activo), haciendo hincapié en el segundo. Para preparar esta última encuesta tomamos las definiciones junto con el contexto de uso que habían obtenido mejores resultados en la encuesta de reconocimiento y planteamos una cuestión de respuesta abierta, pues los informantes debían escribir un refrán que utilizarían en esa situación.

En las tres pruebas realizadas, los informantes encuestados eran hablantes de español como lengua materna. Solicitamos que especificaran su lugar de procedencia con la finalidad de comprobar las realizaciones dialectales que tienen lugar entre los hablantes de lengua española para, después, poder extrapolarlo a los diferentes tipos de estudiantes o aprendientes de español.

Otra de las variables que se tuvo en cuenta fue la edad de los encuestados, segregados en cuatro franjas de edad: "menores de 18 años", considerados menores; "19-35", a los que se les atribuyó la etiqueta de jóvenes; “36-50”, el grupo de adultos; y por último el grupo de "mayores de 50".

En la última encuesta también se recogió información acerca de la frecuencia de uso de las paremias, la forma de aprenderlas y opiniones generales acerca de su uso, como, por ejemplo, si se consideraba que esa paremia era de mala educación.

Las hipótesis con las que partíamos al comenzar nuestro estudio eran las siguientes:

- Se pueden asociar algunas paremias a contextos de uso determinados y, por tanto, dichas paremias pueden ser consideradas pragmatemas.

- La edad influye en el conocimiento paremiológico, siendo los informantes de edad más avanzada los que mejores resultados aportarán.

- El lugar de procedencia de los informantes no influirá en la selección del contexto extralingüístico asociado a las paremias.

\subsection{Análisis y resultados}

Como hemos adelantado, seleccionamos las paremias de uso más frecuente de la prueba piloto:

- Arrieritos somos y en el camino nos encontraremos

- Aunque la mona se vista de seda, mona se queda

- Perro ladrador, poco mordedor

- Quien tiene boca se equivoca

- Quien la sigue la consigue

- A lo hecho, pecho

- Mi gozo en un pozo

- De tal palo, tal astilla

Se ha de señalar que todas estas paremias aparecen en el trabajo de Tarnovska (2005), quien por lo tanto las considera mínimos paremiológicos. 


\subsubsection{Encuesta de reconocimiento}

De las ocho paremias seleccionadas, para la encuesta de reconocimiento se tienen en cuenta solamente las cuatro primeras, quedando las cuatro restantes pendientes de análisis en la última encuesta. Todas ellas son bimembres, es decir, configuradas en una estructura con dos partes diferenciadas.

A continuación, en las Tablas 1-4 se presentan los porcentajes, tanto de la elección del significado concreto extraído de Junceda (1981) y de las respuestas abiertas extraídas de la prueba piloto, como de la preferencia en la forma de uso.

En todos los casos consideramos dos variantes posibles de cada paremia: una forma completa, en la que se reflejaba la paremia en su totalidad, y una forma acortada, que reflejaba únicamente la primera parte de las paremias. Hemos de añadir que la forma acortada en todos los casos puede enunciarse con un patrón entonativo descendente, por lo que lo hemos reflejado con puntos suspensivos. El resultado de estas formas es arrieritos somos, aunque la mona se vista de seda..., perro ladrador... y quien tiene boca...

\begin{tabular}{|l|c|c|c|}
\hline \multicolumn{1}{|c|}{ SIGNIFICADO/ CONTEXTO DE USO } & PORCENTAJE & ACORTADA & COMPLETA \\
\cline { 1 - 2 } $\begin{array}{l}\text { Se está discutiendo con alguien y se le amenaza } \\
\text { con este refrán }\end{array}$ & $59,03 \%$ & & \\
\cline { 1 - 3 } Ha ocurrido algo malo y hay que ser consecuente & $16,87 \%$ & \multirow{2}{*}{$44,6 \%$} & \multirow{2}{*}{$21,69 \%$} \\
\cline { 1 - 2 } $\begin{array}{l}\text { Crees que tienes razón sobre algún tema y los } \\
\text { demás no están de acuerdo }\end{array}$ & $2,41 \%$ & \\
\cline { 1 - 2 } $\begin{array}{l}\text { Se está intentando animar a alguien y le dices esto } \\
\text { para asegurarle que va a ir bien. }\end{array}$ & & & \\
\hline
\end{tabular}

Tabla 1: Arrieritos somos y en el camino nos encontraremos

En la primera tabla se puede apreciar cómo los informantes se decantan en un 59,03\% por la primera definición en la que se especifica que la paremia denota una amenaza en un contexto de discusión, frente al $2 \%$ que apuesta por el contexto contrario, una respuesta más relacionada con un mensaje de ánimo y esperanza al interlocutor.

En cuanto a la forma de expresión, se resuelve un porcentaje equilibrado entre las dos variantes.

\begin{tabular}{|l|c|c|c|}
\hline SIGNIFICADO/ CONTEXTO DE USO & PORCENTAJE & ACORTADA & COMPLETA \\
\cline { 1 - 2 } Tu consideración de algo no va a cambiar & $14,78 \%$ & \multirow{2}{*}{$63 \%$} & $35 \%$ \\
\cline { 1 - 2 } La esencia de algo se mantiene intacta & $44,34 \%$ & & \\
\cline { 1 - 2 } Una persona va a seguir siendo fea & $40,87 \%$ & & \\
\hline
\end{tabular}

Tabla 2: Aunque la mona se vista de seda, mona se queda 
La Tabla 2 refleja porcentajes bastante equilibrados entre dos de las opciones. No obstante, es la definición relativa a la esencia la que acumula mayores respuestas, posiblemente por su neutralidad de contexto.

También se puede observar que más de la mitad de los encuestados prefieren el empleo de la forma acortada a la completa.

\begin{tabular}{|c|c|c|c|}
\hline SIGNIFICADO/CONTEXTO DE USO & PORCENTAJE & ACORTADA & COMPLETA \\
\hline $\begin{array}{l}\text { Se dice de una persona que aparenta mucho, } \\
\text { pero en realidad no es para tanto }\end{array}$ & $13,28 \%$ & \multirow{4}{*}{$33 \%$} & \multirow{4}{*}{$65,3 \%$} \\
\hline $\begin{array}{l}\text { Se dice de una persona que impone mucho en el } \\
\text { desarrollo de una discusión, pero en realidad no } \\
\text { es para tanto }\end{array}$ & $21,83 \%$ & & \\
\hline $\begin{array}{l}\text { Se dice de una persona que realiza muchas } \\
\text { amenazas, pero no las lleva a cabo }\end{array}$ & $40,14 \%$ & & \\
\hline $\begin{array}{l}\text { Te parece una persona que dice muchas cosas, } \\
\text { pero luego no las cumple }\end{array}$ & $24,65 \%$ & & \\
\hline
\end{tabular}

Tabla 3: Perro ladrador, poco mordedor

De nuevo, en la Tabla 3, se presenta un mayor porcentaje en la definición que presenta el refrán como reflejo de una amenaza, con el matiz de que en este caso es solo una aparente amenaza.

A diferencia del caso anterior, se presenta una inclinación a la enunciación en la forma completa. Podría deberse no tanto al desconocimiento del interlocutor de esta expresión, sino a que en la segunda parte del refrán se presenta la parte que otorga el contenido esencial del significado de la paremia.

\begin{tabular}{|l|c|c|c|}
\hline SIGNIFICADO/ CONTEXTO DE USO & PORCENTAJE & ACORTADA & COMPLETA \\
\hline Se usa como consejo & $22,31 \%$ & & \\
\cline { 1 - 2 } $\begin{array}{l}\text { Se usa para defender a alguien que se ha } \\
\text { equivocado }\end{array}$ & $41,98 \%$ & \multirow{2}{*}{$6,9 \%$} & $93 \%$ \\
\cline { 1 - 2 } Se usa como excusa de algo que has hecho mal & $34,71 \%$ & & \\
\hline
\end{tabular}

Tabla 4: Quien tiene boca se equivoca

Por último, en la Tabla 4 destaca el porcentaje de la forma completada de la expresión, posiblemente por denotar la acción a la que hace referencia el significado del refrán.

Entre las dos opciones relacionadas con la defensa a otro o la excusa sobre uno mismo, los informantes prefieren ligeramente la primera, se usa para defender a alguien que se ha equivocado (41,98\%) frente a se usa como excusa de algo que has hecho mal (34,71\%), como se esperaba.

Es también en este caso excepcional en el que se produce la única tendencia notable a la elección de una de las formas de enunciación, decantándose por la forma completa (93 $\%$ de los casos). 
Tras la recopilación de los datos, se realizó el test estadístico chi-cuadrado $(\chi 2)$ para observar el cruce de la variable edad con el significado de los refranes y el contexto de uso, pero en ninguno de los casos se obtuvieron resultados significativos. De esta forma, la hipótesis acerca del cambio de significado en función de la edad quedó rechazada.

Aunque tampoco arrojó resultados estadísticamente significativos, los informantes asociaron mayoritariamente el empleo de paremias a registros informales en los que se producía una relación entre iguales, como la comunicación en un grupo de amigos $(80 \%$ de las respuestas), seguido de la familia (67\%). Se reduce el empleo de refranes $(16 \%)$ en los contextos en los que la jerarquía social distancia a los hablantes, como la relación laboral entre trabajador y jefe.

\subsubsection{Encuesta de acceso}

La tercera encuesta que se realizó se centraba en la disponibilidad paremiológica, por lo que el proceso de adquisición de información fue el contrario al de la encuesta anterior. En esta ocasión, se ofrecía a los informantes la definición más votada en la encuesta de reconocimiento. Dicha definición, además, iba asociada a un acto comunicativo, que delimitamos por introspección. Por último, se ofrecía también un contexto de uso relacionado con los datos extraídos de la primera encuesta. El informante debía escribir la paremia que se emplearía en dicha situación. La Tabla 5 refleja el porcentaje de acierto de cada paremia:

\begin{tabular}{|c|c|c|c|c|}
\hline Paremia & Definición & $\begin{array}{c}\text { Acto } \\
\text { comunicativo }\end{array}$ & $\begin{array}{c}\text { Contexto } \\
\text { extralingüístico }\end{array}$ & Porcentaje \\
\hline $\begin{array}{l}\text { Quien la sigue la } \\
\text { consigue }\end{array}$ & $\begin{array}{l}\text { Alguien ha estado mucho } \\
\text { tiempo intentando algo y por } \\
\text { fin lo consigue }\end{array}$ & Respuesta & $\begin{array}{c}\text { Esperanza } \\
\text { Motivación } \\
\text { Ánimo }\end{array}$ & $84 \%$ \\
\hline A lo hecho, pecho & $\begin{array}{l}\text { Se han tomado malas } \\
\text { decisiones y hay que asumir } \\
\text { las consecuencias }\end{array}$ & Respuesta & $\begin{array}{c}\text { Amenaza } \\
\text { Consecuencia }\end{array}$ & $56 \%$ \\
\hline $\begin{array}{l}\text { Mi gozo en un } \\
\text { pozo }\end{array}$ & $\begin{array}{l}\text { Estabas esperando con muchas } \\
\text { ganas que pasase algo, pero no } \\
\text { ha llegado a ocurrir }\end{array}$ & Enunciación & Decepción & $29 \%$ \\
\hline $\begin{array}{l}\text { De tal palo, tal } \\
\text { astilla }\end{array}$ & $\begin{array}{l}\text { Ha pasado algo y, } \\
\text { sorprendentemente, has } \\
\text { reaccionado igual que tu padre }\end{array}$ & $\begin{array}{l}\text { Respuesta } \\
\text { Incidir en tema } \\
\text { Descripción }\end{array}$ & Comparación & $75 \%$ \\
\hline $\begin{array}{l}\text { Aunque la mona } \\
\text { se vista de seda, } \\
\text { mona se queda }\end{array}$ & $\begin{array}{l}\text { La esencia de algo sigue } \\
\text { intacta aunque se adorne }\end{array}$ & $\begin{array}{l}\text { Respuesta / } \\
\text { Descripción }\end{array}$ & $\begin{array}{l}\text { Crítica a tercera } \\
\text { persona }\end{array}$ & $95 \%$ \\
\hline $\begin{array}{l}\text { Perro ladrador, } \\
\text { poco mordedor }\end{array}$ & $\begin{array}{l}\text { Una persona que suele } \\
\text { amenazar mucho pero luego } \\
\text { no lo lleva a cabo }\end{array}$ & $\begin{array}{l}\text { Respuesta / } \\
\text { Descripción }\end{array}$ & $\begin{array}{c}\text { Crítica a } \\
\text { interlocutor / } \\
\text { Descripción / } \\
\text { Amenaza }\end{array}$ & $93 \%$ \\
\hline $\begin{array}{l}\text { Quien tiene boca } \\
\text { se equivoca }\end{array}$ & $\begin{array}{l}\text { Todos nos equivocamos } \\
\text { alguna vez }\end{array}$ & Respuesta & $\begin{array}{c}\text { Defensa con } \\
\text { comprensión / } \\
\text { Consuelo }\end{array}$ & $45 \%$ \\
\hline
\end{tabular}




\begin{tabular}{|l|l|c|c|c|}
\hline $\begin{array}{l}\text { Arrieritos somos y } \\
\text { en el camino nos } \\
\text { encontraremos }\end{array}$ & $\begin{array}{l}\text { Estás discutiendo con alguien } \\
\text { y lo estás amenazando con } \\
\text { este refrán }\end{array}$ & Respuesta & Amenaza & $29,5 \%$ \\
\hline
\end{tabular}

Tabla 5: Definición de las paremias en relación con sus contextos de uso

Ante las respuestas recogidas, se puede realizar una escala con las frecuencias de acierto de las paremias:

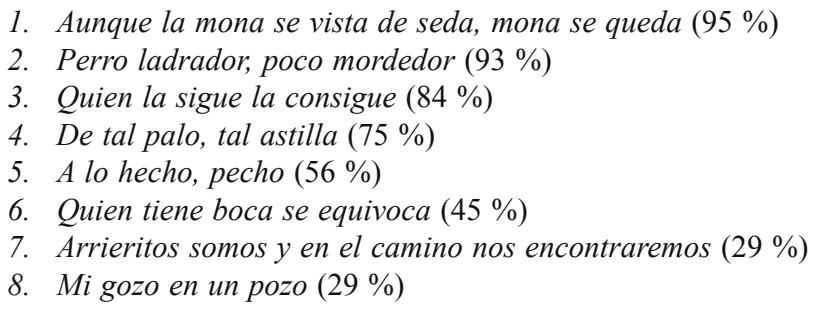

$\mathrm{Al}$ aparecer de forma reiterada asociadas por diferentes hablantes a un contexto comunicativo determinado extraído de su propia interpretación, podría confirmarse que algunas de ellas forman parte del ámbito de los pragmatemas. Para determinar si el reconocimiento de estos contextos extralingüísticos es suficiente para considerarse pragmatema, se toman los porcentajes extraídos de los resultados. Ante la ausencia de trabajos experimentales previos que establezcan criterios de decisión, se decide marcar en un $90 \%$ de acierto, al igual que los porcentajes de significatividad probabilística que marcan la significatividad en una distribución estadística normal. Con este criterio, se establece que pueden considerarse pragmatemas las paremias aunque la mona se vida de seda, mona se queda y perro ladrador, poco mordedor.

Estos resultados también fueron analizados en función de distintas variables cualitativas, entre las que se encuentran la edad, el lugar de procedencia o la frecuencia de uso. En general ninguna aportó resultados significativos exceptuando dos paremias.

El refrán de tal palo, tal astilla arrojó diferencias significativas en función de la edad, $\chi^{2}(6, N=44)=22.69, p<.001$, reflejando que los menores no respondieron con ningún refrán, los jóvenes propusieron el refrán esperado y los adultos y mayores alternaban entre el esperado y otros que guardaban relación con el contexto propuesto, como de padres gatos, hijos misinos. Estos datos permiten observar cómo el conocimiento paremiológico, en cierto modo, está relacionado con la edad, siendo mayor cuanto más avanzada es esta.

También con respecto a este refrán resulta significativo la relación con el lugar de procedencia, $\chi^{2}(8, N=44)=15.28, p=.05$. Esta variable muestra cómo los informantes procedentes de zonas más urbanas, como Madrid o Barcelona, responden con la forma esperada, mientras que los informantes procedentes de otras zonas, como pueblos de Aragón, Castilla La Mancha, o zonas de las Islas Canarias, además de responder el refrán esperado, también mostraron de forma reiterada otras variantes, como bendita la rama que al tronco sale, o de padres gatos, hijos misinos.

El segundo refrán que ofreció datos significativos, en este caso al ser relacionada con la frecuencia de uso, fue arrieritos somos y en el camino nos encontraremos. Como se refleja 
en la Imagen 1, se puede observar claramente la variación que se produce con respecto a la variable. La totalidad de los informantes que consideraron usar refranes siempre en sus conversaciones conocían y usaban este refrán. Aquellos que afirmaron hacer mucho uso de estos presentaron tanto la variante esperada como otras opciones. Hemos de añadir que los otros refranes propuestos, pero en todos los casos, guardaban relación con el contexto propuesto, pues propusieron los refranes ya pedirás agua en el desierto y te daré polvorones, entre otras. Por último, en el caso de aquellos que hacen poco uso de las paremias en la conversación, aumentó el porcentaje de informantes que no supieron qué responder y, en las ocasiones en que respondieron con otro refrán diferente al esperado, este no guardaba relación con el contexto, como lo que no mata engorda.

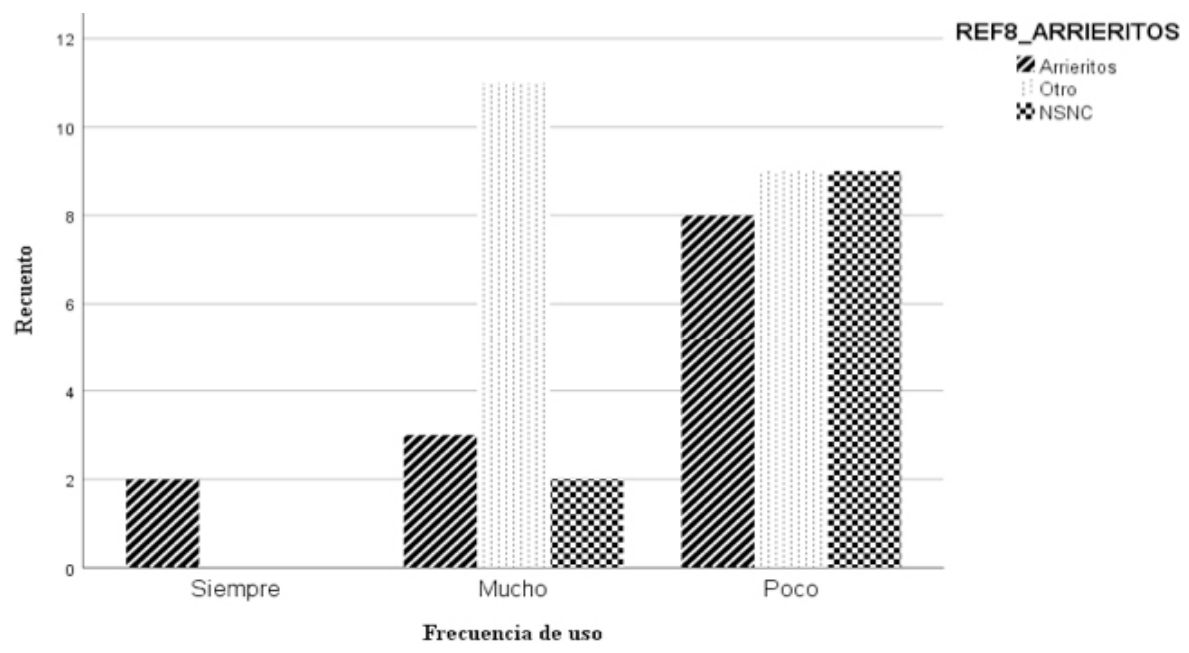

Imagen 1: Representación gráfica de la relación entre la frecuencia de uso y el refrán arrieritos somos y en el camino nos encontraremos

La ausencia de datos significativos en el cruce de variables, en este caso, es un resultado positivo. Si no varía la asociación de los refranes a sus contextos de uso en función de la edad, el lugar de procedencia o la frecuencia de uso de los hablantes, se entiende que, realmente, la relación entre el refrán y su contexto de uso es generalizado y no dependiente de diferentes grupos de hablantes, por lo que se asume que dicha asociación se puede generalizar y, por tanto, enseñar.

Por último, en esta encuesta también se preguntó acerca de las restricciones de uso por la situación comunicativa. Aunque los porcentajes varían, la tendencia es la misma que en la encuesta anterior, siendo el grupo entre iguales el preferido para el empleo de las paremias (84\%), valorado igual que la familia (84\%). De nuevo se restringe su uso en contextos con mayor distancia jerárquica (2\%). No obstante, el $100 \%$ de los informantes aseguraron que no consideraban de mala educación el empleo de paremias en la comunicación. 


\section{DISCUSIÓN Y CONCLUSIÓN}

La Teoría Sentido-Texto permite realizar un análisis de las construcciones fraseológicas basadas en el contexto o la situación extralingüística, una perspectiva diferente al análisis fraseológico tradicional que se ha realizado basado en la tradición literaria o en las variaciones en la construcción de las expresiones. Desde este punto de vista, se ha podido observar que algunas de las paremias analizadas pueden ser consideradas pragmatemas: de una parte hemos encontrado y descrito ciertas restricciones contextuales para cada una de ellas; de otra, dichas restricciones han sido reconocidas por una muestra de hablantes. En este sentido, si las paremias se analizan bajo esta premisa comunicativa, se podría plantear una perspectiva basada en el contexto en las aplicaciones anteriormente citadas y no tanto en su construcción, como se ha venido haciendo hasta ahora, tal como hemos mostrado.

Las principales aplicaciones del análisis del contexto extralingüístico de uso tienen que ver con la enseñanza del español, tanto en la educación secundaria a hablantes nativos de español, como en la enseñanza como lengua extranjera. En ambos casos, se apostaría por el desarrollo de competencias basadas en el saber hacer, es decir, en su integración en la comunicación real. Para ello, será necesario partir de metodologías didácticas que avalen el diseño de actividades que tengan estos objetivos comunicativos como fin, como es el caso del enfoque comunicativo.

Como se ha comprobado, resulta de vital importancia el contexto comunicativo en el que se producen las paremias, por lo que dicho contexto será clave en el proceso de enseñanzaaprendizaje. Si el contexto comunicativo se convierte en el criterio de clasificación de las paremias, tal y como se presentaba en la Tabla 5, a partir de él se pueden plantear actividades relacionadas con la identificación de paremias en textos reales. Estos pueden tomarse de series televisivas o de los medios de comunicación, y se les pueden asociar diferentes contextos de uso o situaciones de la vida cotidiana asociadas a dichas paremias.

Aunque la aproximación realizada en este trabajo desde la Teoría Sentido-Texto ha permitido realizar una primera aproximación empírica a la asociación paremia-contexto extralingüístico de uso, aún queda mucho trabajo por hacer. Por un lado, debemos especificar, en la medida de lo posible, los contextos asociados a las paremias más conocidas por los hablantes que son al mismo tiempo pragmatemas. Por otra parte, debemos comprobar lo que ocurre con las paremias de uso específico, aunque no formen parte del mínimo paremiológico, como es el caso de mierda que no mata engorda, asociada exclusivamente a un contexto en el que ha caído comida al suelo, o en martes ni te cases ni te embarques, considerado como consejo de no realizar ninguna actividad el segundo día de la semana.

\section{REFERENCIAS BIBLIOGRÁFICAS}

Anscombre, J. C. (1997). "Reflexiones críticas sobre la naturaleza y el funcionamiento de las paremias". Paremia, 6, pp. 43-54.

Barrios Rodríguez, M. A. (2017). "Hacia un concepto amplio de pragmatema y sus aplicaciones en ELE. ELE: el caso de iqué + sust./adj.!”. En Almeida Cabrejas, B., Blanco Canales, A., García Sánchez, J. J. y Jiménez López, M. D (eds.). Investigaciones actuales en Lingüistica. Vol. II: Semántica, Morfología y Lexicología. Alcalá de Henares: Servicio de Publicaciones de la Universidad de Alcalá. 
Beghadid, H. M. (2013). "El enfoque comunicativo, una mejor guía para la práctica docente". Actas del IV Taller ELE e interculturalidad del Instituto Cervantes de Oran, pp. 112-120.

Blanco Escoda, X. (2014). "Microestructura lexicográfica para unidades frásticas: los pragmatemas". Káñina, vol. 38, 3, pp. 13-18.

Consejo De Europa (2002): El Marco común europeo de referencia para las lenguas, aprendizaje, enseñanza, evaluación. Madrid: Anaya y CVC. Disponible en http://www.cvc.cervantes.es/obref/ marco [Consulta: 20/07/2021]

Corpas Pastor, G. (1996). Manual de fraseología española. Madrid: Gredos.

Crida Alvarez, C., y Sevilla Muñoz, J. (2015). "La problemática terminológica en los estudios paremiológicos". Anuari de filologia. Estudis de lingüística, 5, pp. 67-77.

Decreto 48/2015, de 14 de mayo, del Consejo de Gobierno, por el que se establece el currículo de la Educación Secundaria Obligatoria para la Comunidad de Madrid. Boletín Oficial de la Comunidad de Madrid, 118.

Díaz Pérez, J. C. (1997). "Desarrollo de la competencia paremiológica en estudiantes de enseñanza primaria y secundaria". Paremia, 6, pp. 189-194.

Doval, G. (1997). Refranero temático español (Palabras mayores). Madrid: Ediciones del Prado.

Fernández Pampín, V. (2012). "Las unidades fraseológicas en la Educación Secundaria. El Refranero multilingüe como punto de partida". Unidades fraseológicas y TIC, 217.

Hymes, D. H., \& Bernal, J. G. (1996). "Acerca de la competencia comunicativa". Forma y función, 9, pp 13-37.

Instituto Cervantes (2007): Niveles de Referencia para el español, Plan Curricular del Instituto Cervantes. Madrid: Biblioteca Nueva.

Junceda, L. (1998). Diccionario de refranes, dichos y proverbios (Diccionario léxico Espasa). Madrid: Espasa-Calpe.

Leal Riol, M. J. (2013). “Estrategias para la enseñanza y aprendizaje de la fraseología en español como lengua extranjera". Paremia, 22, pp. 161-170.

Lomas, C. y Osoro, A. (1994). "Enseñar Lengua". En Lomas, C. y Osoro A. (Comp.) El enfoque comunicativo de la enseñanza de la lengua. Barcelona: Paidós

Mel'čuk, I. (1996). "Lexical functions: A tool for the description of lexical relations in a lexicon". In Wanner, L. Lexical functions in lexicography and natural language processing. Amsterdam/ Philadelphia: John Benjamin, 209-278.

Mel'čuk, I. (2015). "Clichés, an understudied subclass of phrasemes"

Penadés Martínez, M. I. (1998). Materiales para la didáctica de las unidades fraseológicas: estado de la cuestión. REALE, 9-10, pp. 125-145.

Polguère, A. (2011). "Classification sémantique des lexies fondée sur le paraphrasage". Cahiers de Lexicologie, 98, pp. 197-211.

Solano Rodríguez, M. A. (2007): "El papel de la conciencia fraseológica en la enseñanza y aprendizaje de una lengua extranjera", en González Rey, M. I. (ed.), Les expressions figées en didactique des langues étrangères, Cortil-Wodon: EME, pp. 201-211.

Solano Rodríguez, M.A. (2012). "Las unidades fraseológicas del francés y del español: tipología y clasificación”. Paremia, 21, pp. 117-128.

Tarnovska, O. (2005). "El mínimo paremiológico en la lengua española". En Pamies Bertrán, A. Y Luque, J.D. (eds.). La creatividad en el lenguaje: colocaciones idiomáticas y fraseología Granada: Método, pp. 197-217.

Vyshnya, N., Sevilla Muñoz, J, y Gutthy, A. (2007). “Civilización y Cultura”. Eslavística Complutense, $3,7$.

Zuluaga, O. A. (1980). "Introducción al estudio de las expresiones fijas". En Studia Romanica et Lingüística, Vol. 10. Frankfurt. 